\title{
On Certain Eigenspaces of Cographs
}

\author{
Torsten Sander \\ Institut für Mathematik \\ Technische Universität Clausthal \\ D-38678 Clausthal-Zellerfeld, Germany \\ torsten.sander@math.tu-clausthal.de
}

Submitted: Apr 12, 2008; Accepted: Oct 27, 2008; Published: Nov 14, 2008

Mathematics Subject Classification: Primary 05C50, Secondary 15A18

\begin{abstract}
For every cograph there exist bases of the eigenspaces for the eigenvalues 0 and -1 that consist only of vectors with entries from $\{0,1,-1\}$, a property also exhibited by other graph classes. Moreover, the multiplicities of the eigenvalues 0 and -1 of a cograph can be determined by counting certain vertices of the associated cotree.
\end{abstract}

Keywords: cograph, eigenspace, nullity, null space

\section{Introduction}

The class of cographs has can be used to model series-parallel decompositions and, hence, has numerous applications in areas like parallel computing [9] or even biology [6]. Consequently, many research results on cographs have been obtained in recent years (see [2] for an overview). The contribution of the present paper is to study the eigenspaces of cographs for eigenvalues 0 and -1 , namely, to derive the multiplicities of these eigenvalues and to construct particularly simple eigenspace bases.

The particular eigenvalues 0 and -1 play a role in several areas of algebraic graph theory. For example, in the theory of star partitions the eigenvalues 0 and -1 are special cases (cf. [4], chapter 7). Another interesting result is that singular line graphs of trees can be partitioned into two classes, depending on whether a certain graph has either 0 or -1 as a multiple eigenvalue [13].

For easier eigenspace analysis it is often desirable to find a basis for a considered graph eigenspace that is considered structurally "simple". For example, we may require the basis vectors to have entries only from a very restricted set of values. Most notably we are interested in the set $\{-1,0,1\}$, in which case we call the basis simply structured. Several authors have contributed to this topic in recent years (cf. [1], [8], [12], [15]). We show that every cograph admits simply structured eigenspace bases for eigenvalues 0 and 
-1 . These bases can be obtained without solving systems of equations. Further, we study the evolution of these eigenspaces when constructing a cograph by repeated vertex split operations.

\section{Basics and notation}

Let us introduce some notation along with a number of definitions and well-known facts. The symbol $\mathrm{j}$ denotes the all ones vector. Throughout, we assume that graphs are finite, undirected, loopless and simple. We write $N_{G}(x)$ for the neighborhood of the vertex $x$ in $G$, i.e. the set of all vertices of $G$ that are adjacent to $x$.

\subsection{Algebraic graph theory}

Given a graph $G=(V, E)$ with vertex set $V=\left\{x_{1}, \ldots, x_{n}\right\}$ and edge set $E$ we define the $n \times n$ adjacency matrix $A(G)$ of $G$ (with respect to the given vertex order) with entries $a_{i j}=1$ if $x_{i} x_{j} \in E$ and $a_{i j}=0$ otherwise. $\operatorname{By} \operatorname{Eig}(\lambda ; G)$ we denote the eigenspace for eigenvalue $\lambda$ of the matrix $A(G)$. The multiplicity of $\lambda$ equals the dimension of $\operatorname{Eig}(\lambda ; G)$ and is written as $\mu(\lambda ; G)$.

One can interpret eigenvectors of $A(G)$ as vertex weight functions $V \rightarrow \mathbb{R}$, in order to derive a notion of graph eigenvectors that does not depend on the numbering of the vertices. Given an eigenvector $w=\left(w_{i}\right)$ one simply assigns the $i$-th component $w_{i}$ to the $i$-th vertex $v_{i}$ of $G$. Then the vector $w$ is an eigenvector of $G$ for eigenvalue $\lambda$ if and only if the summation rule holds, i.e. for every vertex the sum of the weights of its neighbors equals $\lambda$ times its own weight.

\subsection{Cographs}

For cographs there exist a number of equivalent definitions [2]. For what follows a constructive definition of cographs in terms of split pairs is best suited. Define an operation $O_{1}$ on a vertex of a given graph that splits the vertex i.e. it introduces a new vertex with the same neighborhood. Analogously, an operation $\mathrm{O}_{2}$ splits a vertex in the same manner, but afterwards adds an edge that connects it with the newly created vertex. The class of cographs is defined as all finite graphs that can be obtained from a single vertex by a series of $\mathrm{O}_{1}$ and $\mathrm{O}_{2}$ operations.

In view of the defined operations we call a pair $(u, v)$ of vertices a split pair if their outer neighborhood is the same, i.e. if $N_{G}(u) \backslash\{v\}=N_{G}(v) \backslash\{u\}$. Then a graph is a cograph exactly if it can be reduced to a single vertex by subsequently joining split pairs. This reduction process yields a characteristic series-parallel decomposition tree for every cograph, called cotree.

Every cograph contains at least one split pair, but usually it contains many eligible pairs that can be grouped as follows. Given a graph $G$ with vertex set $V$, we call a set $M \subseteq V$ a module in $G$ if $N_{G}(u) \backslash M=N_{G}(v) \backslash M$ holds for every pair $u$, $v$ from $M$. Consequently, a split pair is a module $M$ with $|M|=2$. A maximum module $M$ 
with $|M| \geq 2$ such that $M$ is an independent set of vertices in $G$ is called an $O_{1}$ cluster. Analogously, if a maximum module $M$ induces a clique in $G$, then we call $M$ an $O_{2}$ cluster. A straightforward argument shows that a vertex cannot be part of more than one cluster.

The number of components of a cograph equals the maximum number of consecutive $O_{1}$ operations at the beginning of its construction. The complement $\bar{G}$ of a cograph $G$ is also a cograph, created by the sequence of exactly the opposite $O_{1}$ and $O_{2}$ operations. Hence, $G$ is connected if and only if $\bar{G}$ is not connected.

\section{Main results}

Let us now determine the multiplicities of the eigenvalues 0 and -1 of a cograph and construct corresponding eigenspace bases. We first cite a theorem about the rank of the adjacency matrix of a cograph:

Theorem 3.1. [3], [10], [14] The rank of a cograph is equal to the number of distinct non-zero rows of its adjacency matrix.

We make use of the previous theorem to state a simply structured basis for the kernel of a cograph:

Theorem 3.2. Let $G$ be a cograph. Then a simply structured basis of $\operatorname{Eig}(0 ; G)$ can be obtained as follows:

1. For every $O_{1}$ cluster $M$ of non-isolated vertices construct $|M|-1$ vectors by assigning weights

- 1 to a fixed vertex of $M$,

- -1 in turn to exactly one other vertex of $M$,

- 0 to all other vertices of $G$.

2. For every isolated vertex create a unit vector what has weight 1 on the respective isolated vertex.

Proof. Since $O_{1}$ clusters cannot overlap the constructed vectors are, obviously, linearly independent. Using the summation rule it is readily verified that all the vectors belong to the kernel of $G$.

Next, observe that the maximal sets of vertices indexed by the redundant rows of the adjacency matrix of $G$ form exactly the $O_{1}$ clusters of $G$ and that the all-zero rows correspond to the isolated vertices. Thus, the basis property of the constructed vectors follows directly from Theorem 3.1.

Corollary 3.3. Let $G$ be a cograph. Let $M_{1}$ be the set of all $O_{1}$ cluster vertices of the connected components of $G$ with at least 2 vertices each and let $m_{1}$ be the number of such clusters. Further, let s denote the number of isolated vertices of $G$. Then,

$$
\mu(0 ; G)=\left|M_{1}\right|-m_{1}+s .
$$


The next theorem reveals a fundamental relation between the kernel of a graph and the eigenspace for eigenvalue -1 of its complement.

Theorem 3.4. [11] Let $G$ be a graph with $n$ vertices. Then,

1. $\operatorname{Eig}(0 ; G) \cap \operatorname{Eig}(-1 ; \bar{G})=\left\{x \in \operatorname{Eig}(0 ; G): \mathrm{j}^{T} x=0\right\}=\left\{x \in \operatorname{Eig}(-1 ; \bar{G}): \mathrm{j}^{T} x=0\right\}$,

2. $|\operatorname{dim} \operatorname{Eig}(0 ; G)-\operatorname{dim} \operatorname{Eig}(-1 ; \bar{G})| \leq 1$,

3. $\operatorname{Eig}(0 ; G) \subset \operatorname{Eig}(-1 ; \bar{G})$ if $\operatorname{dim} \operatorname{Eig}(0 ; G)<\operatorname{dim} \operatorname{Eig}(-1 ; \bar{G})$,

4. $\operatorname{Eig}(0 ; G)=\operatorname{Eig}(-1 ; \bar{G})$ if $\operatorname{dim} \operatorname{Eig}(0 ; G)=\operatorname{dim} \operatorname{Eig}(-1 ; \bar{G})$,

5. $\operatorname{Eig}(0 ; G) \supset \operatorname{Eig}(-1 ; \bar{G})$ if $\operatorname{dim} \operatorname{Eig}(0 ; G)>\operatorname{dim} \operatorname{Eig}(-1 ; \bar{G})$.

For cographs, the eigenspace inclusion relation described in Theorem 3.4 is not arbitrary:

Theorem 3.5. Let $G$ be a cograph. Then, $\operatorname{Eig}(0 ; G) \supseteq \operatorname{Eig}(-1 ; \bar{G})$.

Proof. We proceed by induction over the number of vertices of $G$. For $G=K_{1}$ the result is trivially true since the complement $\bar{G}=K_{1}$ lacks eigenvalue -1 . Let $G$ be a cograph with at least two vertices and assume that the result holds for all cographs with fewer vertices.

If $G$ is disconnected, then by the induction assumption the result holds for each of its components, and hence, by composition, also for $G$ itself.

So let $G$ be connected. We show that for every vector from $\operatorname{Eig}(-1 ; \bar{G})$ the sum over its components vanishes. Then the result follows from Theorem 3.4.

Assume, to the contrary, that there exists a vector $v \in \operatorname{Eig}(-1 ; \bar{G})$ with $\mathrm{j}^{T} v \neq 0$. It follows by Theorem 3.4 that $\operatorname{Eig}(0 ; G) \subseteq \operatorname{Eig}(-1 ; \bar{G})$ so that every vector from the kernel of $G$ has vanishing component sum, in particular the kernel basis vectors of $G$ listed in Theorem 3.2. Hence, according to Theorem 3.4, together with the vector $v$ they form a basis of $\operatorname{Eig}(-1 ; \bar{G})$. Consequently, we may assume without loss that $v$ vanishes on every $\mathrm{O}_{2}$ cluster of $\bar{G}$ (recall that the $O_{2}$ clusters of $\bar{G}$ are the same as the $O_{1}$ clusters of $G$ ), except for at most one vertex per cluster.

If there exists an $O_{2}$ cluster in $\bar{G}$, then it contains a split pair with a vertex $x$ on which $v$ vanishes. Let $G^{\prime}=G \backslash\{x\}$ and let $v^{\prime}=\left.v\right|_{G^{\prime}}$ be the restriction of $v$ to $G^{\prime}$. Then, $\mathrm{j}^{T} v^{\prime} \neq 0$ and $v^{\prime} \in \operatorname{Eig}\left(-1 ; \overline{G^{\prime}}\right)$, contradicting the induction assumption.

If there exists no $O_{2}$ cluster in $\bar{G}$, then $G$ contains no $O_{1}$ cluster but necessarily at least one $\mathrm{O}_{2}$ split pair. Let $G^{\prime}$ be the induced subgraph of $G$ obtained by successively joining $O_{2}$ split pairs for as long as possible. If $G^{\prime}=K_{1}$, then $G$ is a complete graph, for which the result of the theorem is trivially fulfilled. Otherwise, $G^{\prime}$ contains an $O_{1}$ split pair so that $\overline{G^{\prime}}$ contains an $O_{2}$ split pair. Create a vector $v^{\prime}$ that differs from the null vector only on these $\mathrm{O}_{2}$ split pair vertices, where it takes values 1 and -1 . Clearly, $w^{\prime}$ is a valid eigenvector of $\overline{G^{\prime}}$ for eigenvalue -1 . Next observe that, by construction, every vertex of $\bar{G}$ that is not a vertex of $\overline{G^{\prime}}$ is adjacent to either both or none of the 
mentioned $\mathrm{O}_{2}$ split pair vertices. So we can trivially extend $w^{\prime}$ with zeroes to obtain an eigenvector $w$ of $\bar{G}$ for the same eigenvalue since the summation rule still holds. $G$ has no $O_{1}$ cluster and, being connected, no isolated vertices. It follows from Theorem 3.1 that the adjacency matrix of $G$ has full rank and further from Theorem 3.4 that $\operatorname{dim} \operatorname{Eig}(0 ; G)=0$ and $\operatorname{dim} \operatorname{Eig}(-1 ; \bar{G})=1$. We deduce that $w$ must be a multiple of $v$. But $\mathrm{j}^{T} w=0$ and $\mathrm{j}^{T} \neq 0$, so we arrive at another contradiction.

Corollary 3.6. Let $G$ be a cograph. For every $O_{2}$ cluster $M$ construct $|M|-1$ vectors by assigning weights

- 1 to a fixed vertex of $M$,

- -1 in turn to exactly one other vertex of $M$,

- 0 to all other vertices of $G$.

Then the constructed vectors constitute a simply structured basis of $\operatorname{Eig}(-1 ; G)$.

Proof. According to Theorem 3.4 and Theorem 3.5 a basis of $\operatorname{Eig}(-1 ; G)$ is given by taking all vectors of a basis for $\operatorname{Eig}(0 ; \bar{G})$ that have vanishing component sum. Therefore, the result follows from Theorem 3.2.

Corollary 3.7. Let $G$ be a cograph. Let $M_{2}$ be the set of all $\mathrm{O}_{2}$ cluster vertices of $G$ and let $m_{2}$ be the number of such clusters. Then,

$$
\mu(-1 ; G)=\left|M_{2}\right|-m_{2} .
$$

As a consequence of Theorem 3.2 and Corollary 3.6, one can easily construct simply structured eigenspace bases for eigenvalues 0 and -1 for all graphs created during the successive construction of a cograph using operations $O_{1}$ and $O_{2}$. In a sense, the eigenspaces bases evolve during the construction process, by means of embedding and slight modification:

Corollary 3.8. Let $G$ be a cograph and let $B$ be an eigenspace basis for eigenvalue 0 according to Theorem 3.2. Assume that $G^{\prime}$ is obtained from $G$ by $O_{i}$ splitting a vertex $v$ of $G$. Let $v^{\prime}$ be the newly created vertex in $G^{\prime}$. Let $B^{\prime}$ initially consist of all vectors of $B$ embedded into $G^{\prime}$ by setting the weight of $v^{\prime}$ to zero. Transform the set $B^{\prime}$ according to the following rules:

- Case $i=1$ : Choose a vector from $B^{\prime}$ that does not completely vanish on the vertices of the $O_{1}$ cluster that $v^{\prime}$ belongs to. Add a new vector to $B^{\prime}$ that resembles the chosen vector, except that the weights of the -1 vertex and of $v^{\prime}$ have been swapped. If no such vector exists, then add to $B^{\prime}$ a vector that is 1 on $v,-1$ on $v^{\prime}$ and zero on all other vertices.

- Case $i=2$ and $v$ is not an $O_{1}$ cluster vertex in $G$ : Leave $B^{\prime}$ as it is. 
- Case $i=2$ and $v$ belongs to an $O_{1}$ cluster in $G$ : If there exists a vector in $B^{\prime}$ that is 1 on $v$, then choose a fixed vertex $w$ different from $v$ but in the same $O_{1}$ cluster of $G$ and swap the weights of $v$ and $w$ for all vectors in $B^{\prime}$. In any case $B^{\prime}$ now contains exactly one vector that does not vanish on $v$. Remove it from $B^{\prime}$.

Then $B^{\prime}$ is an eigenspace basis $G^{\prime}$ for eigenvalue 0 and coincides with a basis obtainable by application of Theorem 3.2 to $G^{\prime}$.

A similar eigenspace evolution can be outlined for eigenvalue -1 .

From Corollary 3.8 we can directly derive how the multiplicities of the eigenvalues 0 and -1 evolve under successive $O_{1}$ and $O_{2}$ operations. Clearly, every $O_{1}$ splitting of a vertex increases the dimension of $\operatorname{Eig}(0 ; G)$. The dimension of $\operatorname{Eig}(-1 ; G)$ may remain unchanged or drop by one, depending on whether an $O_{2}$ cluster has been "hit":

Corollary 3.9. Let $G$ be a connected cograph with at least two vertices and let $G^{\prime}$ be obtained by a splitting operation on a vertex of $G$. Then the dimensions of the eigenspaces change according to the table below.

\begin{tabular}{|c|c|c|c|}
\hline oper. & dest. vertex type & $\mu\left(0 ; G^{\prime}\right)-\mu(0 ; G)$ & $\mu\left(-1 ; G^{\prime}\right)-\mu(-1 ; G)$ \\
\hline \hline$O_{1}$ & non $O_{2}$ cluster & 1 & 0 \\
\hline$O_{1}$ & $O_{2}$ cluster & 1 & -1 \\
\hline$O_{2}$ & non $O_{1}$ cluster & 0 & 1 \\
\hline$O_{2}$ & $O_{1}$ cluster & -1 & 1 \\
\hline
\end{tabular}

As remarked in section 2.2, the construction process of a cograph can be described by a series parallel decomposition tree, namely, its cotree. The vertices of the cograph form the leaves of the (rooted and directed) cotree. Every non-leaf vertex of the cotree is labelled type 1 or 2, depending on the operation used to obtain its children.

The cotree $T$ of a cograph $G=(V, E)$ can be obtained in $O(|V|+|E|)$ time, cf. [5], [7]. Using the cotree $T$, it is straightforward to compute the multiplicities of eigenvalues 0 and -1 of $G$. Observe that two vertices of $G$ belong to the same cluster (or to the set of isolated vertices) exactly if the corresponding leaves of $T$ have a common parent. Therefore, the clusters and isolated vertices can be determined by a depth first search starting from the root of $T$. The respective contributions of the vertices to $\mu(0 ; G)$ and $\mu(-1 ; G)$ can be calculated using Corollaries 3.3 and 3.7. As a result, we obtain the following algorithm: 
Algorithm 3.10. Let $T$ be the cotree of a connected cograph $G$. If $r$ is the root of $T$, then $(\mu(0 ; G), \mu(-1 ; G))=$ getMult $(r)$, using the procedure given below.

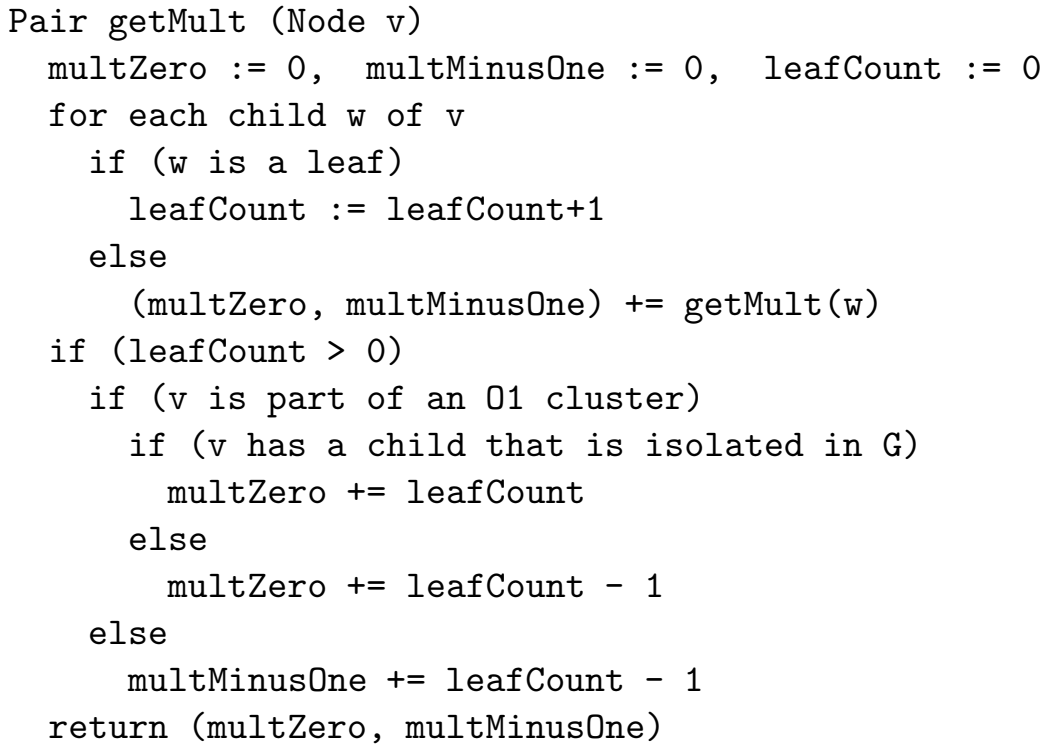

\section{Conclusion}

We have studied the eigenspaces for the eigenvalues 0 and -1 of cographs and shown that their multiplicities can be determined by simply counting certain types of cotree vertices. We have further given constructive proof that for every cograph there exist bases of these eigenspaces that consist only of vectors with entries from $\{0,1,-1\}$. Finally, using simple transformations it is possible to evolve eigenspace bases with entries from $\{0,1,-1\}$ along with the construction of a cograph from a single vertex by repeated vertex splitting operations.

Finally, note that the inclusion relation given in Theorem 3.5 is very remarkable. Exactly the same property is also exhibited by forests [11]. It would be interesting to find other common graph classes for which this property holds.

\section{References}

[1] Akbari, S., Alipour, A., Ghorbani, E., And Khosrovshahi, G. $\{-1,0,1\}$ basis for the null space of a forest. In Linear Algebra Appl. (2006), vol. 414, pp. 506-511.

[2] Brandstädt, A., Le, V. B., And Spinrad, J. P. Graph classes: a survey. SIAM Monographs on Discrete Mathematics and Applications. Society for Industrial and Applied Mathematics (SIAM), Philadelphia, PA, 1999.

[3] Chang, G. J., Huang, L.-H., And YeH, H.-G. On the rank of a cograph. In Linear Algebra Appl. (2008), vol. 429, pp. 601-605. 
[4] Cvetković, D., Rowlinson, P., And Simić, S. Eigenspaces of graphs, vol. 66 of Encyclopedia of Mathematics and its Applications. Cambridge University Press, Cambridge, 1997.

[5] Damiand, G., Habib, M., And Paul, C. A simple paradigm for graph recognition: application to cographs and distance hereditary graphs. In Theoret. Comput. Sci. (2001), vol. 263, pp. 99-111.

[6] Gagneur, J., Krause, R., Bouwmeester, T., and Casari, G. Modular decomposition of protein-protein interaction networks. In Genome Biology (2004), vol. 5:R57.

[7] Habib, M., And Paul, C. A new vertex splitting algorithm for cograph recognition. RR-123800, Rapports internes et prépublications du LaBRI, Univ. Bordeaux, 2000.

[8] Hazama, F. On the kernels of the incidence matrices of graphs. In Discrete Math. (2002), vol. 254, pp. 165-174.

[9] Nakano, K., Olariu, S., And Zomaya, A. A time-optimal solution for the path cover problem on cographs. In Theor. Comput. Sci. (2003), vol. 290, pp. 1541-1556.

[10] Royle, G. F. The rank of a cograph. In Electron. J. Comb. (2003), vol. 10, pp. 1-7.

[11] SAnder, T. Inclusion relations of certain graph eigenspaces. Rocky Mt. J. Math. (to appear).

[12] Sander, T., And Sander, J. W. On simply structured kernel bases of unicyclic graphs. In AKCE J. Graphs. Combin. (2007), vol. 4, pp. 61-82.

[13] Sciriha, I. The two classes of singular line graphs of trees. In 5th Workshop on Combinatorics (Messina, 1999), Rend. Sem. Mat. Messina Ser. II (1999), vol. 5, pp. 167-180.

[14] Türker, B. Graph Laplacians and Nodal Domains. Ph. D. Thesis, Universität Wien, Austria, 2003.

[15] Villarreal, R. H. Rees algebras of edge ideals. In Commun. Algebra (1995), vol. 39, pp. 3513-3524. 